\title{
Parental Acceptance-Rejection as Predictor of Alexithymia among Students in Gilgit-Pakistan
}

\author{
S. Hussain and Z. Ahmed
}

\begin{abstract}
This research was conducted to study the alexithymia in association of parental acceptance-rejection among student population in Gilgit-Pakistan. A total of 116 students (male $95 \&$ female 21 ) were recruited from different educational institutes of Gilgit city. The Toronto Alexithymia Scale was administered to participants along with short forms of the Adult Parental Acceptance-Rejection Questionnaires for mothers and for fathers. Results showed that perceived paternal and maternal acceptance were significantly correlated with the students' alexithymia. Additionally, regression analysis indicated that both perceived paternal and maternal acceptance independently predicted students' alexithymia.
\end{abstract}

Index Terms-Parental acceptance-rejection, students, paternal acceptance, maternal acceptance, alexithymia.

\section{INTRODUCTION}

The word alexithymia is coined by Peter Sifneos in 1972 to describe the condition, characterized by an individual's inability to understand his/her experiences feelings and lack of words to describe these feelings to other. Alexithymia is disturbance in emotional processing can be conceptualized by difficulty in identifying and verbalizing feelings. It remain unchanged therefore, it represents constant trait and positively with neuroticism [1], [2]. When someone cannot express negative feelings will have trouble discharging and neutralizing these feelings either physiologically or psychologically. Ultimately, all feelings either normal or pathological become bodily feelings and appear in somatic complaints.

Researchers have identified various factors as an etiology of alexithymia. Developmental perspective focuses on the importance of various developmental stages. In this process one cannot deny the important and the critical role of the primary caregiver of the growing child. According to this perspective the child's parents may have failed to sufficiently encourage a language of feelings or emotional trauma at any stage of the development, may account for the development of alexithymia [3]. Children's affective expressions were associated with the amount of information, level of intensity, and types of emotion shared in their family [4]. Expression of emotions is a learning process like what to feel and how to express what they have felt in their family [5], [6]. When the expression of affects is constricted in families, children tended to show constricted affect. In contrast when families have high level of expressiveness,

Manuscript received September 20, 2013; revised November 28, 2013.

The authors are with the Behavioural Sciences Department, Karakoram International University, University Road, Gilgit-15100, Pakistan (Correspondence author: Dr. Sadiq Hussain; e-mail: sadiq.hussain@kiu.edu.pk, zubair.ahmed@kiu.edu.pk). their children display higher levels of unrestricted expression [7].

Family variables like positive family expressiveness and negative family expressiveness explained moth of the variance associated with the alexithymia and its sub-factors, which are difficulty identifying feelings and difficulty describing feelings respectively. Additionally, most of the variance in predicting alexithymia was explained by a lack of perceived healthy expressive family environment and social support from family. In conclusion participants from more dysfunctional families that are characterized by lack of positive family expressiveness and social support from family, tended to have higher level of alexithymia. In contrast participants with healthy family environment background that is higher levels of social support from family and positive family expressiveness tended to have lower level of alexithymia [8].

Maternal and paternal parenting styles were moderately associated with alexithymia among psychiatric outpatients [9]. Parental attitude, perceived lack of maternal care, perceive lack of warmth relationship with father, paternal indifference and maternal abuse, and attachment during childhood were associated with alexithymia and tended to play a vital role in the development of alexithymia characteristics during the resting period [10]-[15]. According to the findings of meta-analysis there was a significant negative association between maternal care and alexithymia. The negative correlations effect size between maternal care and difficulty identifying feelings was ranger from small to medium, between maternal care and difficulty describing feelings was ranged from medium to large, and between maternal care and externally oriented thinking was small. The correlation effect size between maternal overprotection and alexithymia was also significant. The relationship effect size between maternal overprotection and difficulty identifying feelings and difficulty describing feelings was range from small to medium but the correlation effect size between maternal overprotection and externally oriented thinking was insignificant. Regarding paternal behavior, there was a significant association between paternal care and alexithymia but the correlation effect sizes between paternal care and two sub-domains of alexithymia which are difficulty identifying feelings and externally oriented thinking were insignificant. Additionally, the correlations effect size between paternal overprotection and alexithymia was also significant. The correlation effect sizes between paternal overprotection and two sub-domains of alexithymia which are difficulty identifying feelings and difficulty describing feelings were significant but small.However, the association between paternal overprotection and externally oriented thinking was 
insignificant. The overall results indicated that both domains of parental (maternal \& paternal) behaviors-parental care and parental overprotection- are significantly associated with total score of alexithymia [16].

\section{A. Research Objectives}

Keeping in mind the above literature, this research was designed to explore the importance of parental behavior in alexithymia among student population of Gilgit-Baltistan, Pakistan. Research findings would work as simulation for research and clinical practice for clinical psychologists, psychologists, and counselors in this impoverished region of Pakistan.

\section{B. Research Hypotheses}

The following hypotheses were formulated to test in this study;

- Maternal behavior would predict students' alexithymia

- Paternal behavior would predict students' alexithymia

\section{Methodology}

\section{A. Participants}

A total of 116 students (male $95 \&$ female 21$)-31$ (27\%) from under matric program, $54(46 \%)$ from intermediate program, and 31 (27\%) university students- were recruited to conduct this research. Their age was ranged from 14-28 years with a mean of 19 years and SD 2.7.

\section{B. Measures}

Demographic information from: this form was used to collect the personal information of participants like gender, age, class of study and so forth.

Adult Parental Acceptance-Rejection Questionnaire: Father-Short Form by Rohner [17]. This is a four-point liker type questionnaire, consist of four sub-scales namely; warmth/affection, hostility/aggression, indifference neglect, and undifferentiated rejection. It measures father behavior as perceived by his offspring.

Adult Parental Acceptance-Rejection Questionnaire: Mother-Short Form by Rohner [17].This is a four-point liker type questionnaire, consist of four sub-scales namely; warmth/affection, hostility/aggression, indifference neglect, and undifferentiated rejection. The questionnaire assesses maternal behavior as perceived by her offspring.

Toronto Alexithymia Scale (TAS-20) by Bagby, Parker, and Taylor [18]. The TAS-20 has three subscales; difficulty describing feelings, difficulty identifying feelings, and externally oriented thinking.

The scale demonstrates good level of psychometric properties. Its internal consistency (Cronbach's alpha) is .81, and test-retest reliability .77 ( $p<.01)$. Regarding the validity, TAS-20 demonstrates adequate levels of convergent and concurrent validity. Additionally, the three factor structure was found to be theoretically congruent with the alexithymia construct.

\section{Procedure}

Before conducting the research, researchers have taken institutional consent from various academic institutions of Gilgit city to collect the research data. After institutional consent, all scales were administered to only consented students in the classroom setting. All scales were scored according to the manual instructions. Predictive analytic software was use to analyze the data.

\section{RESULTS}

Both descriptive and inferential statistical techniques were used to analyze the data. Table I, shows the descriptive statistics results for all administered scales.

TABLE I: DESCRIPTIVE STATISTIC RESUlt FOR ALEXITHYMIA, PARQMOTHER, AND PARQ-FATHER

\begin{tabular}{lcc}
\hline \hline Scales & Mean & SD \\
\hline TAS-20 & 53.8 & 6 \\
PARQ-Mother & 44.2 & 8 \\
PARQ-Father & 43.4 & 9 \\
\hline \hline
\end{tabular}

Table II, represents the correlations between alexithymia and maternal acceptance-rejection and paternal acceptancerejection. The relationships between alexithymia and maternal acceptance-rejection and paternal acceptance rejection are statistically significant. It indicates that when parental (maternal \& paternal) rejection increases the level of alexithymia also increases.

TABLE II: CORRELATIONS FOR ALEXITHYMIA, PARQ-MOTHER, AND PARQ-FATHER

\begin{tabular}{|c|c|c|c|}
\hline Scales & 1 & 2 & 3 \\
\hline 1. TAS-20 & & & \\
\hline 2. PARQ-Mother & $21 *$ & & \\
\hline 3. PARQ-Father & $22 *$ & $51^{* *}$ & \\
\hline
\end{tabular}

Additionally, we have evaluated the perceived parental (maternal \& paternal) acceptance-rejection as possible predictor of alexithymia as a shown in Table III. Both variables (maternal \& paternal acceptance-rejection) are significant predictors of alexithymia. Perceived maternal acceptance-rejection explained $4 \%$ variance and perceived paternal acceptance-rejection explained $5 \%$ variance in alexithymia.

TABLE III: REGRESSION RESULT SUMMARY FOR ALEXITHYMIA AS RESPONSE AND PARENTAL (MATERNAL \& PATERNAL) ACCEPTANCEREJECTION AS PREDICTORS

\begin{tabular}{lccccc}
\hline \hline Scales & \multicolumn{7}{c}{ Standardized Coefficients } & & \\
\hline & $\mathrm{R}$ & $\mathrm{R}^{2}$ & Beat & $t$ & $\mathrm{Sig}$ \\
\hline PARQ-Mother & 21 & .04 & 21 & 2.3 & .02 \\
PARQ-Father & 22 & .05 & 22 & 2.4 & .01 \\
\hline \hline
\end{tabular}

\section{DISCUSSION}

This research was conducted to study the parental behavior as possible predictor of alexithymia among students in Gilgit-Baltistan, Pakistan. The research findings indicate that parental (both maternal \& paternal) behavior is a significant predictor of alexithymia. Emotional expression patterns of parents such as blunted, inconsistent, aversive, and indifference to the emotional communication of young children lead them to become insecurely attached and unable to develop the full capacity for emotional communication. Theses parental responses toward children's expression of emotions, can affect children's understanding of emotion, style of emotional expression, 
and their ability to regulate emotion. When children fail to understand and express their emotions, alexithymia may develop [19].

Positive self-expressivity in the family environment was negatively correlated with alexithymia and its subcategories which are difficulty identifying feelings, difficulty describing feelings, and externally oriented thinking, while lack of expressiveness, higher level of negative selfexpressivity in the family, and perceived lack of social support from family were positively associated with alexithymia. High level of negative expressiveness within the family environment may be associated with the psychiatric patients' tendency to be alexithymic and alexithymia was predicted by emotional expressiveness of family. Yong adults with alexithymia tended to be brought up in families where there was poor affective expressiveness Adult university students' alexithymia was predicted by the extent to which their family members were permitted and encouraged them to express their opinions and emotions to each other during their childhood [20]-[24]. Reference [25], [26] described the characteristics of the mother of alexithymia patients as sedative rather than satisfying mothers. Such mothers neglect the psychic needs of their children. Reference [27] claimed that alexithymia is resulted from the empathetic failure on the part of the parents to respond to their child's need for validating the mirroring of his mental productions and acceptance of his idealizing projections.

Alexithymia total score and difficulty identifying feelings and difficulty describing feelings were correlated with childhood emotional and physical abuse among primarycare patients. Adults with alexithymia have reported histories of childhood physical or sexual abuse [28]-[31].

\section{CONCLUSION}

In the light of our findings and other related empirical evidences which described alexithymia in terms of early childhood experiences, particularly focused on parenting, it can be concluded that parental behavior during childhood is an important and crucial factor in the development of alexithymia in later adult life.

\section{REFERENCES}

[1] M.-S. Francisco, A.-G. Manuel, and O.-S. Beatriz, "Alexithymia state or trait?" The Spanish Journal of Psychology, vol. 6, pp. 51-59, May 2003.

[2] R. Pandeyand M. K. Mandal, "Eysenckian personality dimensions and alexithymia: Examining the overlap terms of perceived autonomic arousal," Personality and Individual Differences, vol. 20, pp. 499-504, April 1996.

[3] R. J. Muller, "When a patient has no story to tell: Alexithymia," Psychiatric Times, vol. 17, pp. 1-6, July 2000.

[4] P. Bronstein, M. Fitzgerald, M. Briones, J. Pieniadz, and A. D'Ari, "Family emotional expressiveness as a predictor of early adolescent social and psychological adjustment," The Journal of Early Adolescence, vol. 13, pp. 448-471, November1993.

[5] A. R. Hochschild, "Emotion work, feeling rules, \& social structure," American Journal of Sociology," vol. 85, pp. 551-575, November 1979.

[6] C. Saarni, "Children's understanding of display rules for expressive behavior," Developmental Psychology,vol. 15, pp. 424-429, July 1979.

[7] G. J. Taylor, R. M. Bagby, and J. D. A. Parker, "Disorders of affect regulation," Alexithymia in Medical and Psychiatric Illness, Cambridge: Cambridge University Press, 1999.
[8] C. Pope, "Family of origin correlates with alexithymia," Ph.D. dissertation, B. A. Hendrix College, The University of Central Arkansas, 2007.

[9] C. G. Kooiman, S. V. R. Vellinga, P. Spinhoven, N. Draijer, R. W. Trijsburg, and H. G. Rooijmans, "Childhood adversities as risk factors for alexithymia and other aspects of affect dysregulation in adulthood," Psychother Psychosom, vol. 73, pp. 107-116, March 2004.

[10] C. G. Kooiman, P. Spinhoven, R. W. Trijsburg, and H. G. Rooijmans, "Perceived parental attitude, alexithymia and defense style in psychiatric outpatients," Psychother Psychosom, vol. 67, pp. 81-87, 1998.

[11] C. D. Panfilis, P. Rabbaglio, C. Rossi, G. Zita, and C. Maggini, "Body image disturbance, parental bonding and alexithymia in patients with eating disorders," Psychopathology,vol. 36, pp. 239-46, October 2003.

[12] O. Mason, M. Tyson, C. Jones, and S.Potts, "Alexithymia: its prevalence and correlates in a British undergraduate sample," Psychology and Psychotherapy: Theory, Research and Practice, vol. 78, pp. 113-25, March 2005.

[13] A. D. Rick and S. Vanheule, "The relationship between perceived parenting, adult attachment style and alexithymia in alcoholic inpatients," Addictive Behaviors, vol. 31, pp. 1265-1270, July 2006.

[14] F. P. Gil, M. Weigl, T. Wessels, D. Irnich, E. Baumuller, and A Winkelmann, "Parental bonding and alexithymia in adults with fibromyalgia," Psychosomatics, vol. 49, pp. 115-122, March-April 2008.

[15] I. Fukunishi, H. Sei, Y. Morita, and R. H.Rahe, "Sympathetic activity in alexithymics with mother's low care," Journal of Psychosomatic Research, vol. 46, pp. 579-589, June 1999.

[16] F. A. Thorberg, R. M. Young, K. M. Sullivan, and M. Lyvers, "Parental bonding and alexithymia: A meta-analysis," European Psychiatry, vol. 26, pp. 187-193, April 2011.

[17] R. P. Rohner and A. Khaleque, "Parental acceptance-rejection questionnaires (PARQ): Test Manual," in Handbook for the Study of Parental Acceptance and Rejection, $4^{\text {th }}$ ed., R. P. Rohner and A. Khaleque, CT: Rohner Research Publications, 2005, pp. 187-225.

[18] R. M. Bagby, J. D. A. Parker, and G. J. Taylor, "The twenty-item Toronto Alexithymia Scale-I. Item selection and cross-validation of the factor structure," Journalof Psychosomatic Research, vol. 38, pp. 23-32, January 1994.

[19] P. M. Crittenden, "Peering into the black box: An explanatory treatise on development of self in young children," in Rochester Symposium on Developmental Psychopathology, Disorders and Dysfunctions of the Self, D. Cicchetti, and S. L. Toth, Eds., Rochester, NY: Rochester University Press, 1994, vol. 5, pp. 79-158.

[20] P. Yelsma, A. J. Hovestadt, W. T. Anderson, and J. E. Nilsson, "Family-of-origin expressiveness: Measurement, meaning, and relationship to alexithymia," Journal of Marital and Family Therapy, vol. 26, pp. 353-363, July 2000.

[21] P. Yelsma, A. J. Hovestadt, J. E. Nilsson, and B. D. Paul, "Clients' positive and negative expressiveness within their families and alexithymia," Psychological Reports, vol. 82, pp. 563-569, April 1998.

[22] S. J. Pollak, R. M. Beren, and J. E. Freeman, "Alexithymia among orthodox Jews: The role of object relations, family emotional expressiveness, and the presence of a disabled sibling," Disability Studies Quarterly, vol. 28, 2008.

[23] H. Berenbaum and T. James, "Correlates and retrospectively reported antecedents of alexithymia," Psychosomatic Medicine, vol. 56, pp. 353-359, July-August 1994.

[24] S. Kench and H. Irwin, "Alexithymia and childhood family environment," Journal of Clinical Psychology, vol. 56, pp. June 737$745,2000$.

[25] J. McDougall, "The psychosoma and the psychoanalytic process," International Review of Psychoanalysis, vol. I, pp. 437-459, 1974.

[26] J. McDougall, "A child is being eaten I: Psychosomatic states, anxiety neurosis and hysteria-a theoretical approach: II: the abysmal mother and the cork child a clinical illustration," Contemporary Psychoanalysis, vol. I, no. 6, pp. 417-459, October 1980.

[27] H. Kohut and E. Wolf. "The disorders of the self and their treatment: An outline," International Journal of Psychoanalysis, vol. 59, pp. 413-426, 1978

[28] J. Matti, S. Luutonen, H. V. Reventlow, P. Patterson, H. Karlsson, and R. K. R. Salokangas, "Alexithymia and childhood abuse among patients attending primary and psychiatric care: Results of the RADEP survey," Psychosomatics, vol. 49, pp. 317-325, July-August 2008.

[29] D. Scherand J. A. Twaite, "The relationship between child sexual abuseand alexithymic symptoms in a population of recovering adult substance misusers," Journal of Child Sexual Abuse, vol. 8, pp. 25-40, 1999. 
[30] H. Berenbaum, "Childhood abuse, alexithymia and personality disorder," Journal of Psychosomatic Research, vol. 41, pp.585-95, December 1996.

[31] C. Zlotnick, M. T. Shea, T. Pearlstein, E. Simpson, E. Costello, and A. Begin, "The relationship between dissociative symptoms, alexithymia, impulsivity, sexual abuse, and self-mutilation," Comprehensive Psychiatry, vol. 37 pp. 12-16, January 1996.

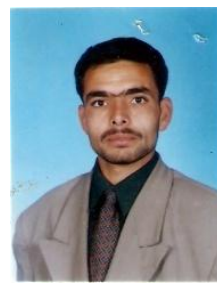

S. Hussain was born in 1983 in a small valley (Nalter valley) of Gilgit city Pakistan. From Federal Government high school for boys Nomal, he has done the matriculation with science in 1998. From Federa Government Degree College for Boys, Gilgit, he has earned the bachelor's degree in Arts in 2004. From University of Karachi, he has done Masters in psychology in 2006. He has earned his PhD. Degree from Institute of Clinical Psychology, University of

Karachi in January 2012.

After Ph.D. in Clinical Psychology, he has joined Karakoram International University as assistant professor at Department of Behavioural Sciences, Karakoram International University Gilgit, Pakistan.His research interest focuses on parenting and its consequences on offspring's psychological adjustment, academic achievement, mental health and so for. Following are his research recent publication: 1) S. Hussain, T. Alvi, A. Zeeshan, and S. Nadeem. "Perceived Childhood Paternal AcceptanceRejection Among Adults," Journal of the College of Physicians and
Surgeons Pakistan, vol. 4, pp. 269-271, 2013. 2) S. Hussain, and S. Munaf. "Gender difference in perceived childhood father rejection and psychological adjustment in adulthood," Journal of Behavioral Sciences [Special Issue: Parental Acceptance-Rejection in the Pakistani Context], vol. 22, pp.100-114, 2012. 3) Dr. Hussain is member of International Society for Interpersonal Acceptance and Rejection and Pakistan Association of Clinical Psychologists.

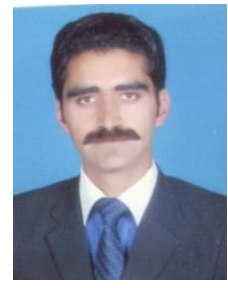

Z. Ahmed was born in 1986 in Gilgit city Pakistan. From Sir Syed Public shclool Gilgit, he has done the matriculation with science in 2001 and from Gate Way International Academy Abbottabad he has done his higher secondary degree. He has earned the bachelor's degree in management sciences (BBA) from Sarhad university of science and technology Peshawar Pakistan in 2006. From Quaid-i-Azam University Islamabad, he has done Masters in Anthropology in 2009. He has earned his M.phil Degree from Quaid-i-Azam University in 2012.

After M.phil in Anthropology he has joined different National and International development organizations working in Pakistan.

He has joined Karakoram International University in 2012 as a lecturer at Department of Behavioural Sciences, Karakoram International University Gilgit, Pakistan. His research interest focuses on social, Psychological and cultural issues around. 